\title{
Aseptic cavernous sinus thrombosis after internal carotid arterial occlusion in polycythaemia vera
}

\author{
ELDAD MELAMED, ELIEZER A. RACHMILEWITZ, \\ AVINOAM RECHES, AND SYLVAN LAVY \\ From the Departments of Neurology and Haematology, Hadassah University Hospital \\ and Hebrew University-Hadassah Medical School, Jerusalem, Israel
}

SYNOPSIS Two patients with untreated polycythaemia vera developed intracranial internal carotid arterial occlusion followed by monocular blindness and the characteristic features of ipsilateral cavernous sinus thrombosis. Primary septic source and signs of systemic infection were absent. It is suggested that the predisposing factors in this unusual syndrome were hyperviscosity and venous sludging induced by the basic haematological disorder and progression of the thrombotic process within the internal carotid artery towards its intracavernous portion with occlusion of the ophthalmic artery and of the arterial branches which supply the walls of the sinus.

Cavernous sinus thrombosis is almost invariably septic and is usually associated with a primary infective source in the face, throat, mouth, ear, mastoid or in the sphenoidal, ethmoidal, or maxillary sinuses (Clune, 1963). Aseptic cavernous sinus thrombosis is a rare occurrence and has been described in association with head injury, surgical procedures of the fifth nerve, and carcinomatous invasion of the base of the skull (Grove, 1936; Boniuk, 1972; Smith, 1974). In the following paper two patients with previously unrecognized polycythaemia vera are presented in whom aseptic cavernous sinus thrombosis developed after ipsilateral internal carotid arterial occlusion.

\section{CASE 1}

A 44 year old asthmatic man with no history of hypertension, diabetes, or heart disease had a sudden onset of left hemiparesis and hemihypaesthesia $2 \frac{1}{2}$ years before the present hospitalization. Haemoglobin was $17.5 \mathrm{~g} / \mathrm{dl}$ and the haematocrit $56 \%$-findings which were attributed at that time to his lung disease. A right carotid angiogram was normal and disclosed no narrowing or occlusion of the extra- or intracranial arteries. The neurological symptoms and signs gradually subsided within three weeks and the patient remained with no

(Accepted 20 October 1975.) residual deficit. The patient was readmitted because of a three day history of progressive weakness and $\Omega$ sensory change in the left limbs. On admission, the blood pressure was $110 / 70 \mathrm{mmHg}$, the pulse rate was $80 /$ minute and regular, and the temperature $36.7^{\circ} \mathrm{C}$. There was marked plethora. Few expiratory wheezes were heard over both lungs. The spleen was palpated $2 \mathrm{~cm}$ below the left costal margin. Physical examination was otherwise normal. The patient was alert and oriented with no speech difficulties. There was no nuchal rigidity. Carotid pulses in the neck were equally palpable with no bruits. There was slight weakness of the left limbs and lower portion of the face. Deep tendon reflexes were hyperactive, the plantar response extensor, and the tone mildly increased on the left. Touch and pain sensations were reduced in the left limbs with impaired stereognosis in the left hand. The pupils were equal and reacting. Eye movements, visual acuity, and the funduscopic examination were normal. Weakness of the left limbs rapidly progressed during the first 36 hours after admission and the left upper extremity became completely paralysed.

Laboratory examinations revealed: haemoglobin $18.0 \mathrm{~g} / \mathrm{dl}$; red blood cells (RBC) $6.5 \cdot 10^{6} \cdot \mathrm{mm}^{-3}$; haematocrit $58 \%$; white blood cells (WBC) $15.6 \cdot 10^{3} \cdot \mathrm{mm}^{-3}$ with a normal differential count; platelets $560 \cdot 10^{3} \cdot \mathrm{mm}^{-3}$; blood volume $86.3 \mathrm{ml} / \mathrm{kg}$ (upper normal limit $80 \mathrm{ml} / \mathrm{kg}$ ); red cell mass $38 \mathrm{ml} /$ $\mathrm{kg}$ (upper normal limit $33 \mathrm{ml} / \mathrm{kg}$ ); serum uric acid $476 \mu \mathrm{mol} / 1$ and total bilirubin $22.2 \mu \mathrm{mol} / 1$; vitamin $\mathrm{B}_{12} 1000 \mu \mathrm{g} / \mathrm{l}$; and folic acid $260 \mu \mathrm{g} / \mathrm{l}$. Urinalysis, and 320 
serum urea nitrogen, glucose, electrolytes, liver function tests, total glycerides, calcium, and phosphorus were within normal limits; serum protein electroand immunoelectrophoresis, antinuclear factor, cryoglobulin and cryofibrinogen, and VDRL test were either normal or negative. Lumbar puncture yielded clear, colourless, cerebrospinal fluid under normal pressure with no cells and with normal protein and glucose levels. The electrocardiogram and skull and chest radiographs were normal. Arterial blood gases levels were normal. The electroencephalographic recording disclosed moderate slowing of activity over the right parietotemporal regions. Brain scan $\left({ }^{99 \mathrm{~m}} \mathrm{Tc}\right)$ revealed an area of increased uptake of isotope in the right parietal region. Bilaterally equal retinal pressures were found in the ophthalmodynamometric examination. A right brachial angiogram, performed 36 hours after admission, disclosed complete occlusion of the intracranial portion of the internal carotid artery, immediately above the origin of the ophthalmic artery.

Seven days after admission, the patient complained of pain behind and around the right eye and blurring of vision in the same eye. Rapidly progressive right ocular proptosis with severe chemosis and periorbital oedema developed during the next 24 hours. Excessive lacrimation, redness, and congestion of conjunctival vessels were noted. Visual acuity deteriorated to complete right mono-ocular blindness with inability to distinguish between light and darkness. Complete ptosis of the right upper lid was observed. Extraocular movements were first limited and later absent in the distribution of the third nerve. Subsequently, abduction of the eye was also lost with the ensuing complete external ophthalmoplegia. The right pupil became dilated, fixed and unresponsive to light. Touch and pain sensations were found to be reduced in the distribution of the first division of the fifth nerve on the right. Corneal sensation was likewise lost. There were no pulsations and bruits over the proptosed eyeball. Funduscopy disclosed marked venous congestion and several retinal haemorrhages, with no papilloedema. The findings were confined to the right eye. There were no fever, chills, nausea, vomiting, or signs of meningeal irritation throughout the course of the disease. There was no clinical or laboratory evidence of infective focus in the face, mouth, throat, ears, mastoids, air sinuses, or elsewhere. Radiographs of the skull, base of skull, sinuses, and mastoids were normal, as was the repeated lumbar tap. Cerebral phlebograms were not performed.

The patient was treated with repeated phlebotomies, heparin first in large and later in low doses, and melphalan (4-6 $\mathrm{mg}$ per week). A slow pro- gressive improvement was noted in the ocular manifestations. Pain, lacrimation, proptosis, periorbital oedema, conjunctival injection, and venous congestion gradually receded. Visual acuity improved and gradually came back to normal. Eye movements were regained, first in abduction and later in the distribution of the third nerve. Ptosis receded and the pupil gradually returned to mid-position and became responsive to light. Funduscopic examination disclosed disappearance of venous congestion. Complete ophthalmological recovery occurred within three weeks. The left-sided weakness and sensory impairment began to improve almost simultaneously and in six weeks cleared completely. There was no neurological or ocular deficit on follow-up examinations for one year, and his present blood count is within normal limits.

\section{CASE 2}

A 43 year old Arab male had a sudden onset of weakness of the left limbs, four months before admission. There was no history of diabetes mellitus, hypertension, or heart disease. The hemiparesis gradually improved and the patient remained with minor residual deficit manifested as mild weakness of the left hand. A month before admission there was an abrupt exacerbation of left-sided weakness. A week later there was a sudden and complete loss of vision in the right eye. After another week the patient developed rapidly progressive right ocular proptosis with swelling of periorbital tissues, increased lacrimation, and severe pain behind and around the right eyeball. There were no fever, chills, nausea, or vomiting. On admission, the blood pressure was $140 / 80 \mathrm{mmHg}$, the pulse rate was $80 /$ minute and regular, and the temperature $36.5^{\circ} \mathrm{C}$. The patient was plethoric with mild acrocyanosis. Few inspiratory and expiratory râles were heard over both lungs. The spleen was palpated $4.0 \mathrm{~cm}$ below the left costal margin. There was no sign of an infective focus in the head or elsewhere. The patient was alert, cooperative, and well-oriented with no speech impairment. The neck was supple and the carotid pulses were equally palpable with no bruits. There was mild weakness of the left limbs, more marked in the upper one. Deep tendon reflexes were hyperactive and the plantar response was extensor on the left. There was no facial weakness or sensory impairment. There was complete right monocular blindness with inability to distinguish between light and darkness. The right eye was severely proptosed with marked oedema of the periorbital tissues and excessive lacrimation. The conjunctiva was red, chemotic, with severe venous congestion. There were no pulsations or bruits in the right eye. Ptosis was 


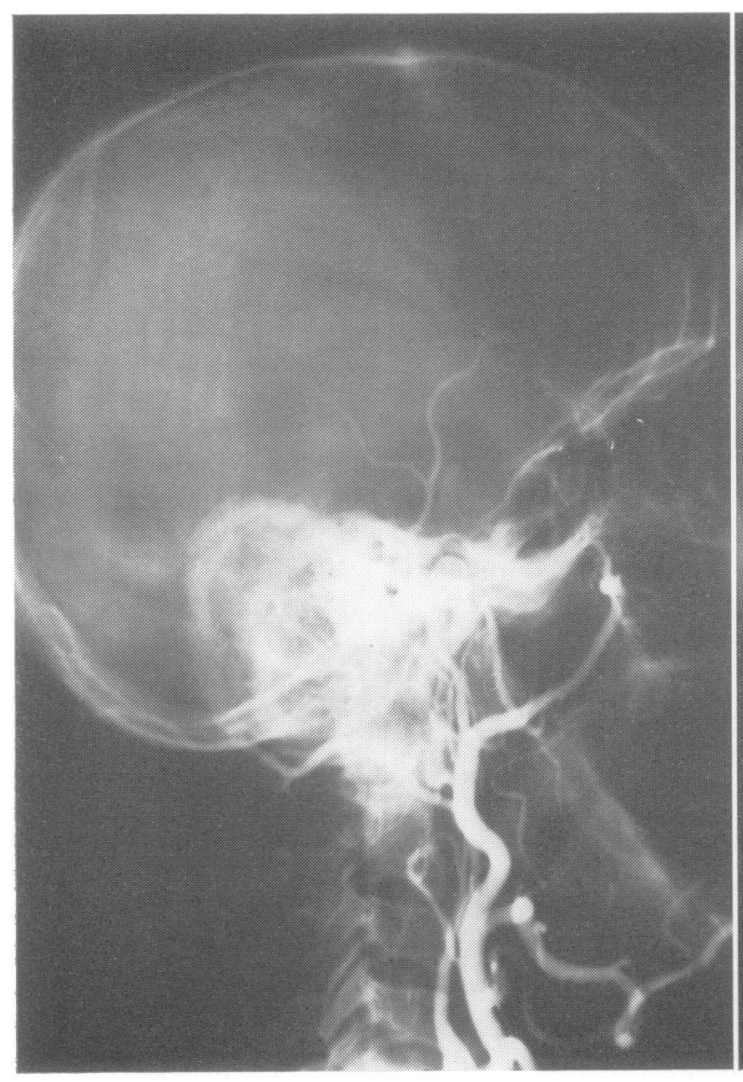

(a)

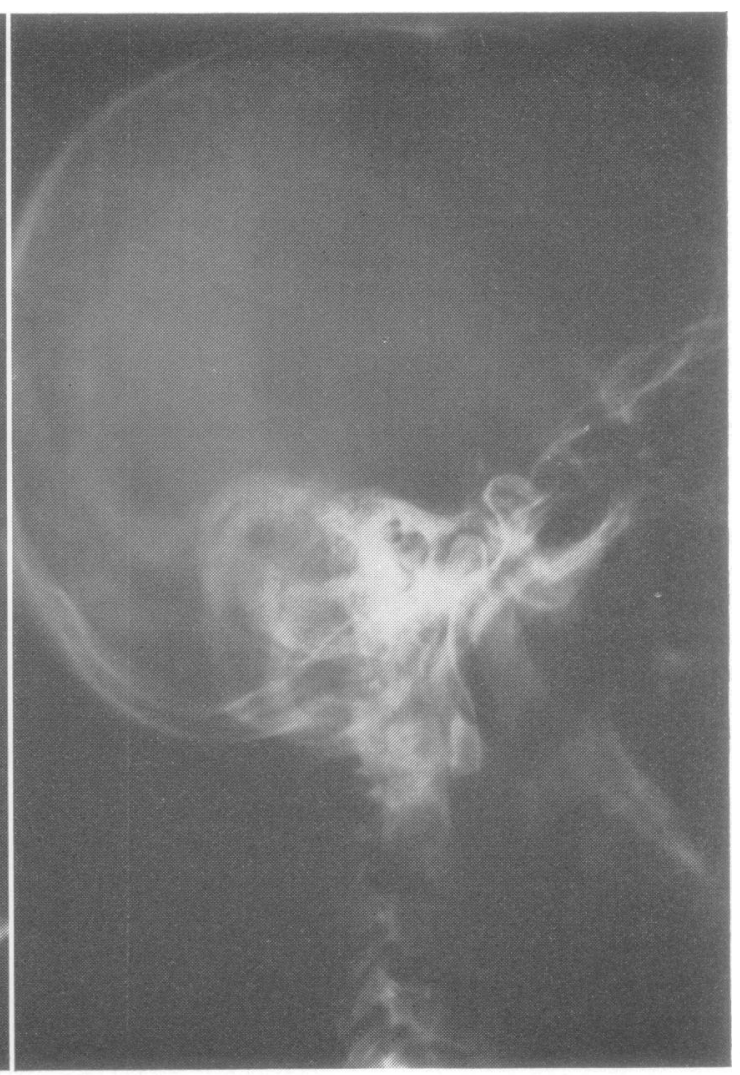

(b)

FIGURE Case 2. Right brachial angiogram, lateral view: (a) two seconds after injection, showing slow and sludged flow in the extracranial portion of the internal carotid artery. (b) 10 seconds after injection, showing complete occlusion at the intracavernous portion of the internal carotid artery.

observed in the right upper lid. There was marked paresis of the right internal rectus and less of the superior rectus muscle. Other eye movements were present. The right pupil was maximally dilated, fixed, and did not react to light. Right corneal sensation was absent. Funduscopic examination disclosed closure of the central retinal artery and a cherry-red macula, retinal oedema, focal haemorrhages mainly along veins, and severe venous congestion in the right eye. The left eye remained normal throughout the disease course.

Laboratory examinations revealed: haemoglobin $21.3 \mathrm{~g} / \mathrm{dl}$; haematocrit $71 \%$, RBC $8.4 \cdot 10^{6} \cdot \mathrm{mm}^{-3}$; WBC $24 \cdot 10^{3} \cdot \mathrm{mm}^{-3}$ with normal differential count; platelets $130 \cdot 10^{3} \cdot \mathrm{mm}^{-3}$; blood volume $114 \mathrm{ml} / \mathrm{kg}$; red cell mass $78 \mathrm{ml} / \mathrm{kg}$; serum uric acid $595 \mu \mathrm{mol} / 1$ and the total bilirubin $20.5 \mu \mathrm{mol} / \mathrm{l}$; vitamin $\mathrm{B}_{12} 620$ $\mu \mathrm{g} / \mathrm{l}$; folic acid $390 \mu \mathrm{g} / \mathrm{l}$; serum iron $12.53 \mu \mathrm{mol} / \mathrm{l}$. Urinalysis, and serum urea nitrogen, glucose, electrolytes, liver function tests, total glycerides, calcium, and phosphorus were within normal limits; serum protein electro- and immuno-electrophoresis, the antinuclear factor and VDRL test were negative or normal. Cerebrospinal fluid was normal. The electrocardiogram and radiography of the chest, skull, base of skull, mastoids, maxillary, sphenoidal, and ethmoidal sinuses were normal, as were the electroencephalographic recording and the brain scan. A right brachial angiogram disclosed complete occlusion of the intracavernous portion of the internal carotid artery (Figure). Cerebral phlebograms were not performed.

The patient was treated with repeated phlebotomies, heparin, first intravenously in large doses and 
later subcutaneously in low doses, melphalan, and allopurinol. Slow and gradual improvement was observed during the course of four weeks. Proptosis, periorbital oedema, chemosis, lacrimation, ocular pain, and conjunctival and retinal venous congestion receded and later cleared completely. The right pupil returned to the normal position with good reaction to light. Left hemiparesis improved though residual slight weakness of the left hand remained. Vision in the right eye was regained, though lower nasal quadrantanopsia persisted on follow-up examinations for six months. His blood count is within normal limits.

\section{DISCUSSION}

Both patients developed the typical features of unilateral cavernous sinus thrombosis after ipsilateral internal carotid arterial occlusion. The constellation of symptoms and signs and the course of the disease seem to rule out orbital cellulitis, abscess or tumour, retro-orbital space occupying lesion such as sphenoidal ridge meningioma, and malignant exophthalmos as possible causes of the ocular involvement. The absence of pulsations and bruits over the affected eyes and the angiographic findings exclude the possibility of carotid-cavernous fistula. In addition, it is unlikely that the arterial occlusion, per se, was responsible for the entire clinical picture. The sinus thrombosis in these patients was considered as aseptic, since there had been no evidence of a primary infective source and signs of systemic infection were absent.

Mathew et al. (1971) demonstrated internal carotid arterial narrowing and occlusion in four patients with septic cavernous sinus thrombosis. The arterial lesions were attributed to internal carotid arteritis induced by the septic process within the sinus. Thus, in two of the cases, the symptoms and signs referable to the arterial involvement occurred 10 and 11 days respectively after the onset of sinus thrombosis, whereas none developed in the others. The situation is altogether different in our patients, since, in both, symptoms and signs of internal carotid arterial occlusion and cerebral ischaemia preceded those of sinus thrombosis by 10 and 14 days respectively. In addition, evidence of a previous, transient cerebrovascular event in the distribution of the same internal carotid arterial system was present in both patients.

The sequence of events suggests that the sinus thrombosis did not develop independently but that it might have been closely related to the preceding arterial occlusion. Factors which presumably played a role in this unusual association may be suggested. The two patients had polycythaemia vera on the basis of several criteria. The spleen was enlarged in both. A significant increase was found in RBC, WBC, and platelet counts (the latter only in the first case) with hyperuricaemia. The blood volume and red cell mass were elevated. This underlying haematological disorder was unrecognized and untreated before the development of the cerebral and ocular manifestations. Polycythaemia vera is well known to be associated frequently with thrombotic cerebrovascular events (Lawrence $e t$ al., 1953; Millikan et al., 1960) and most probably played a major role in the pathogenesis of arterial occlusion in these patients. Conceivably, the polycythaemia with the resultant blood hyperviscosity contributed to venous sludging and induced or precipitated the aseptic thrombotic process within the cavernous sinus.

To our knowledge, neither polycythaemia vera nor pre-existing internal carotid arterial occlusion have been previously reported in association with cavernous sinus thrombosis. It seems, therefore, that an additional factor, closely related to the particular anatomical setting of the process, might have been involved. As it traverses the cavernous sinus, the internal carotid artery gives off branches which supply the walls of the sinus (Gray, 1959). The ophthalmic artery arises from the internal carotid artery just as this vessel is leaving the cavernous sinus. Both patients developed ipsilateral blindness as part of the syndrome. Monocular loss of vision may occur during the course of cavernous sinus thrombosis but it is usually a late complication (Smith, 1974). It may develop after internal carotid arterial occlusion if the lesion is below the origin of the ophthalmic artery. However, blindness in the first patient developed simultaneously with the symptoms and signs of cavernous sinus thrombosis and not immediately with those of cerebral ischaemia. Cerebral angiography, performed before appearance of the ocular manifestations, disclosed complete 
occlusion of the internal carotid artery immediately above the origin of the ophthalmic artery. Loss of vision in the second patient occurred seven days after the onset of signs of cerebral ischaemia and seven days before those of cavernous sinus thrombosis. Cerebral angiography, performed when the patient had already developed all the ocular manifestations, showed complete occlusion of the intracavernous portion of the internal carotid artery. Thus, it may be postulated that in both patients the primary site of internal carotid occlusion was above the origin of the ophthalmic artery. The thrombotic process later progressed towards the intracavernous portion of the internal carotid artery occluding during its propagation the orifices of the branches leading to the walls of the cavernous sinus. In the absence of adequate collateral circulation, this might have led to cessation of blood flow through the ophthalmic artery with the resultant retinal ischaemia and monocular blindness. Likewise, ischaemic changes might have occurred in the walls of the cavernous sinus, thereby predisposing to its thrombosis.

It is reasonable to assume that the pathogenetic mechanism underlying this unusual syndrome involved a combination of these predisposing factors. In both patients the cerebral and ocular manifestations subsided after anticoagulant therapy and specific treatment for the polycythaemia vera. The clinician should be aware that aseptic cavernous sinus thrombosis may be a complication of internal carotid artery occlusion, especially if other precipitating factors such as polycythaemia vera are present.

\section{REFERENCES}

Boniuk, M. (1972). The ocular manifestations of ophthalmic vein and aseptic cavernous sinus thrombosis. Transactions of the American Academy of Ophthalmology and Otolaryngology, 76, 1519-1534.

Clune, J. P. (1963). Septic thrombosis within the cavernous chamber. Review of the literature with recent advances in diagnosis and treatment. American Journal of Ophthalmology, 56, 33-39.

Gray, H. (1959). Anatomy of the Human Body, 27th edn, pp. 633-634. Edited by C. M. Goss. Lea and Febiger: Philadelphia.

Grove, W. E. (1936). Septic and aseptic types of thrombosis of the cavernous sinus. Archives of Otolaryngology, 24, 29-50.

Lawrence, J. H., Berlin, N. I., and Huff, R. L. (1953). The nature and treatment of polycythemia. Studies in 263 patients. Medicine, 32, 323-388.

Mathew, N. T., Abraham, J., Taori, G. M., and Gopalakrishna, V. I. (1971). Internal carotid artery occlusion in cavernous sinus thrombosis. Archives of Neurology (Chic.), 24, 11-16.

Millikan, C. H., Siekert, R. G., and Wishnant, J. P. (1960). Intermittent carotid and vertebral basilar insufficiency associated with polycythemia. Neurology (Minneap.), 10, 188-196.

Smith, B. H. (1974). Infections of the dura and its sinuses. In Clinical Neurology, vol. 2, pp. 15-16. Edited by A. B. Baker and L. H. Baker. Harper and Row: Hagerstown, ? Maryland. 